\title{
Optimización del desempeño del observador al medir la presión arterial en el consultorio: declaración de posición de la Comisión Lancet de Hipertensión*
}

\author{
Raj Padwal, ${ }^{1}$ Norm R. C. Campbell, ${ }^{2}$ Aletta E. Schutte, ${ }^{3}$ Michael Hecht Olsen, ${ }^{4}$ Christian Delles, ${ }^{5}$ \\ Anthony Etyang, ${ }^{6}$ J. Kennedy Cruickshank, ${ }^{7}$ George Stergiou, ${ }^{8}$ Michael K. Rakotz, ${ }^{9}$ Gregory Wozniak, ${ }^{9}$ \\ Marc G. Jaffe, ${ }^{10}$ Ivor Benjamin, ${ }^{11}$ Gianfranco Parati ${ }^{12}$ y James E. Sharman ${ }^{13}$
}

Forma de citar Padwal R, Campbell NRC, Schutte AE, Olsen MH, Delles C, Etyang A, et al. Optimización del desempeño del observador al medir la presión arterial en el consultorio: declaración de posición de la Comisión Lancet de Hipertensión. Rev Panam Salud Publica. 2020;44:e88. https://doi.org/10.26633/RPSP.2020.88

RESUMEN

La hipertensión arterial es una causa modificable muy prevalente de enfermedades cardiovasculares, accidentes cerebrovasculares y muerte. Medir con exactitud la presión arterial es fundamental, dado que un error de medición de $5 \mathrm{mmHg}$ puede ser motivo para clasificar incorrectamente como hipertensas a 84 millones de personas en todo el mundo. En la presente declaración de posición se resumen los procedimientos para optimizar el desempeño del observador al medir la presión arterial en el consultorio, con atención especial a los entornos de ingresos bajos o medianos, donde esta medición se ve complicada por limitaciones de recursos y tiempo, sobrecarga de trabajo y falta de suministro eléctrico. Es posible reducir al mínimo muchos errores de medición con una preparación adecuada de los pacientes y el uso de técnicas estandarizadas. Para simplificar la medición y prevenir errores del observador, deben usarse tensiómetros semiautomáticos o automáticos de manguito validados, en lugar del método por auscultación. Pueden ayudar también la distribución de tareas, la creación de un área específica de medición y el uso de aparatos semiautomáticos o de carga solar. Es fundamental garantizar la capacitación inicial y periódica de los integrantes del equipo de salud. Debe considerarse la implementación de programas de certificación de bajo costo y fácilmente accesibles con el objetivo de mejorar la medición de la presión arterial.

Palabras clave Presión arterial; equipos de medición; consenso; salud mundial; hipertensión arterial; oscilometría.

* Traducción oficial al español del artículo original en inglés efectuada por la Organización Panamericana de la Salud. En caso de discrepancia, prevalecerá la versión original en inglés. Wolters Kluwer Health, Inc. y sus sociedades no se hacen responsables de la exactitud de la traducción del original inglés ni de los eventuales errores que esta pueda contener.

Cita del artículo original: Padwal R, Campbell NRC, Schutte AE, Olsen MH, Delles C, Etyang A, et al. Optimizing observer performance of clinic blood Pressure measurement: a position statement from the Lancet Commission on Hypertension Group. J Hypertens. 2019:37(9):1737-45. doi: https://doi.org/ 10.1097/HJH.0000000000002112

1 Departamento de Medicina, Universidad de Alberta, Edmonton (Canadá); 2 Departamento de Medicina, Fisiología y Farmacología y Salud Comunitaria, Instituto O'Brien de Salud Pública e Instituto Cardiovascular Libin de Alberta, Universidad de Calgary, Calgary, Alberta (Canadá); ${ }^{3}$ Equipo de Investigación de la Hipertensión en Africa (HART), Unidad de Investigación MRC: Hipertensión y Enfermedades Cardiovasculares, Universidad del Noroeste, Potchefstroom (Sudáfrica); ${ }^{4}$ Departamento de Medicina Interna, Hospital de Holbæk, Dinamarca; y Centro de Medicina Individualizada en Enfermedades Arteriales
(CIMA), Hospital Universitario de Odense, Universidad del Sur de Dinamarca, Odense (Dinamarca); ${ }^{5}$ Instituto de Ciencias Cardiovasculares y Médicas, Universidad de Glasgow (Reino Unido); ${ }^{6}$ Programa de Investigación KEMRI-Fundación Wellcome, Kilifi (Kenya): ${ }^{7}$ Escuela de Ciencias de la Nutrición y del Curso de la Vida, King's College, Hospitales St. Thomas \& Guy, Londres (Reino Unido); ${ }^{8}$ Centro de Hipertensión STRIDE- ${ }^{7}$, Universidad Nacional y Capodistríaca de Atenas, Facultad de Medicina, Departamento de Medicina III, Hospital Sotiria, Atenas (Grecia); ${ }^{9}$ Asociación Médica Estadounidense (AMA), Chicago (Estados Unidos de América); ${ }^{10}$ Iniciativa de Estrategias Vitales "Resolve to Save Lives", Nueva York (Estados Unidos de América); y Centro Médico Kaiser Permanente de South San Francisco (Estados Unidos de América); ${ }^{11}$ Asociación Estadounidense del Corazón (AHA), Centro Cardiovascular, Facultad de Medicina de Wisconsin, Wauwatosa (Estados Unidos de América); ${ }^{12}$ Departamento de Medicina y Cirugía, Universidad de Milán-Bicocca, Milán (Italia); e Instituto Auxológico Italiano, IRCCS, Departamento de Ciencias Cardiovasculares, Neurales y Metabólicas, Hospital S. Luca, Milán (Italia); ${ }^{13}$ Instituto Menzies de Investigación Médica, Universidad de Tasmania, Hobart (Australia).

$\bowtie$ Raj Padwal, rpadwal@ualberta.ca 


\section{INTRODUCCIÓN}

En todo el mundo, la hipertensión arterial (HTA) es el principal factor de riesgo modificable de muerte y discapacidad; afecta a unos 1400 millones de adultos y causa más de 10 millones de muertes cada año [1,2]. Es una de las principales causas de cardiopatías, accidentes cerebrovasculares e insuficiencia renal crónica, así como uno de los principales factores que contribuyen a aumentar los costos de salud [3]. Su prevalencia a nivel mundial es alta, del $31 \%$ en adultos [2]. Sin embargo, en cifras absolutas, los países de ingresos bajos o medianos son los que registran la mayor carga de enfermedad, con más de mil millones de personas afectadas y niveles de conocimiento, tratamiento y control muy por debajo de los existentes en los países de ingresos altos [2]. En consecuencia, los esfuerzos actuales para mejorar el diagnóstico, la prevención, el tratamiento y el control de la HTA a nivel mundial deben incluir intervenciones adaptadas que prioricen la reducción de las disparidades regionales [4].

La medición exacta y fiable de la presión arterial (PA) es esencial para el diagnóstico y el tratamiento adecuados de la HTA [5]. A nivel poblacional, una diferencia de $5 \mathrm{mmHg}$ en la PA sistólica corresponde a una variación absoluta del $6 \%$ y relativa del $30 \%$ en la prevalencia de HTA [6]. En consecuencia, el efecto de un error de $5 \mathrm{mmHg}$ en la medición de la PA, con base en una prevalencia mundial de 1400 millones [2], podría ocasionar que se clasifiquen incorrectamente como hipertensas a 84 millones de personas en todo el mundo. Por consiguiente, las consecuencias de la medición inexacta son graves.

La PA es un parámetro fisiológico que cambia en forma constante como respuesta a factores endógenos y estímulos exógenos [7]. Esta variabilidad hace difícil evaluar la PA "habitual", definida como la PA real o genuina de una persona. Medir la PA es quizás el procedimiento más frecuente en la clínica médica y, aunque a primera vista parece sencillo, en realidad son muchos los pasos que deben realizarse de forma secuencial y en condiciones óptimas para obtener un resultado reproducible que refleje la PA habitual. En consecuencia, la persona responsable de medir la PA, que en adelante llamaremos "observador", debe seguir meticulosamente las siguientes técnicas recomendadas [8]. Riva-Rocci et al. [9] reconocieron hace más de un siglo las dificultades que plantea la naturaleza variable de la PA; estos autores señalaron que obtener múltiples mediciones y estandarizar las condiciones de medición podrían optimizar el uso de la técnica en la práctica clínica. En su inspirador artículo escrito en 1896 llegaron a la conclusión que, "si se descuidan los procedimientos y el médico se conforma con una lectura grosera, el método se volverá inútil y será abandonado rápidamente como un mero capricho de científicos" [9].

Lamentablemente, en la práctica clínica contemporánea la medición de la PA se realiza a menudo de manera deficiente, y este tipo de medición no estandarizada de la PA lleva a errores que pueden alterar de forma inadecuada las decisiones terapéuticas en un $20 \%$ a $45 \%$ de los casos [10-13]. El problema de la medición no estandarizada de la PA persiste desde hace décadas, pese a la exhaustiva formación y a los esfuerzos sustanciales por concientizar sobre las consecuencias adversas de medir la PA en el consultorio de forma inexacta $[5,14]$. Este error obedece en gran medida a las limitaciones de tiempo y a una técnica deficiente que lleva a realizar mal la auscultación [10,15]. A continuación, se analizan algunas posibles soluciones para reducir al mínimo el error, como simplificar el proceso de medición usando tensiómetros automáticos y alentar a los observadores a que reciban capacitación certificada y periódica para promover el uso constante de técnicas estandarizadas de medición.

El objetivo de la Comisión Lancet de Hipertensión fue establecer las acciones clave para mejorar el manejo de la PA en todo el mundo, tanto a nivel poblacional como individual, y elaborar una campaña para la adopción en los países, de las acciones sugeridas con el objeto de reducir la repercusión de la HTA [4]. La finalidad de la presente declaración de posición es brindar orientación para optimizar el desempeño relacionado con el observador al medir la PA en el consultorio para diagnóstico y tratamiento de la HTA, con especial foco en los países de ingresos bajos o medianos. Se presenta inicialmente un repaso breve de las diferentes modalidades de medición, incluido un comentario sobre la técnica óptima y los errores derivados de cualquier desviación de las prácticas estandarizadas de medición. A continuación se describe en qué medida repercute la capacitación del observador en la medición de la PA. Luego se aborda la medición de la PA en el entorno de los países de ingresos bajos o medianos y, más concretamente, las condiciones que impiden lograr la mejor práctica. Por último, se presentan las recomendaciones para optimizar la exactitud del observador al momento de medir la PA en el consultorio y se proponen sugerencias para el futuro.

\section{Tipos de medición de la presión arterial usados en la práctica clínica y técnica óptimade medición}

La medición directa (intraarterial) e indirecta (con manguito o brazalete) de la PA son los dos métodos principales empleados en el entorno asistencial; se presentan de forma resumida en el cuadro 1. La medición indirecta suele llevarse a cabo mediante auscultación o con algún tensiómetro semiautomático o automático, por lo general con la técnica oscilométrica (cuadro 1). Aunque en la bibliografía existen diferentes definiciones de medición semiautomática y automática de la PA, para el presente artículo consideramos "semiautomáticos" los tensiómetros que requieren inflado a mano (p. ej., comprimiendo una perilla de goma). Una vez completado el inflado, estos aparatos suelen tener un mecanismo automático de desinflado para determinar la PA. Los aparatos plenamente automáticos, en cambio, tienen automatizados tanto el inflado como el desinflado: el usuario se limita a iniciar una medición (por lo general, pulsando un botón) y el resto del proceso de inflado y desinflado es automático. La medición automática de la PA en el consultorio (MAPAC) es un subtipo de medición automática que implica la obtención de mediciones secuenciales en lugar de una sola toma. En la técnica oscilométrica, primero se detectan, se filtran y se procesan los pulsos arteriales y, a continuación, se aplica un algoritmo patentado para calcular la PA [16].

La medición de la PA debe hacerla de forma meticulosa un observador capacitado siguiendo un método estandarizado (cuadro 2 y figura 1; figuras complementarias S1-S5, http:/ / links.lww .com/HJH/B93) [5]. El promedio de múltiples mediciones correctas ya sea por la técnica auscultatoria o, más recientemente, por la oscilométrica automática, en el transcurso de dos o más consultas clínicas, fue el método usado para calcular la PA habitual en la mayoría de los estudios pronósticos 

claramente detectables: la aparición y desaparición de los ruidos de Korotkoff, respectivamente. No obstante, rara vez se hace bien en la práctica clínica, lo cual es una limitación importante.

Puede realizarse con un esfigmomanómetro de mercurio, aneroide o electrónico. El mercurio se ha prohibido en muchas jurisdicciones, y los esfigmomanómetros aneroides contienen piezas móviles que requieren calibración frecuente que a menudo no se hace, con lo que la exactitud disminuye con el paso del tiempo.

La medición auscultatoria simultánea con dos observadores a ciegas, realizada según los métodos estandarizados con un esfigmomanómetro de mercurio y cámara inflable del tamaño adecuado, es el método de referencia para los estudios de validación de PA, pero es demasiado engorrosa para la práctica clínica.

Semiautomático 0 automático
Ambos métodos suelen usar la técnica oscilométrica, aunque en raras ocasiones un tensiómetro totalmente automático puede emplear la auscultación electrónica o ambos. Los tensiómetros semiautomáticos no se usan a menudo, pero pueden resultar ventajosos en el ámbito de los países de ingresos bajos o medianos porque el inflado se hace a mano (y no necesitan, pues, una fuente de alimentación). El desinflado semiautomático está controlado por el propio aparato. Los tensiómetros automáticos controlan tanto el inflado como el desinflado.

Una cuestión importante con los tensiómetros automáticos es que muchos de los comercializados no tienen una validación adecuada en cuanto a la exactitud de la medición.

Las principales ventajas de la medición automática de la PA, que la convierten en la técnica preferida para el consultorio, son la simplificación del proceso de medición y la eliminación de los errores de auscultación relacionados con el observador. La medición automática de la PA en el consultorio (MAPAC) permite obtener múltiples mediciones secuenciales en ausencia de observación (lo cual reduce el "efecto de la bata blanca"), pero estos tensiómetros son relativamente costosos y pueden requerir más espacio y tiempo en la consulta clínica; hace falta más investigación para definir su papel. Fuera del consultorio, las modalidades automáticas de medición ambulatoria y domiciliaria de la PA proporcionan mejores prestaciones diagnósticas y pronósticas con respecto a las mediciones en el consultorio, incluida la detección de la HTA de bata blanca y la HTA enmascarada. Si los recursos lo permiten, se recomienda usar la MAPAC y la medición ambulatoria o domiciliaria de la PA.

Una desventaja es que la técnica oscilométrica calcula de forma indirecta la PA (esto es, no existe ningún fenómeno fisiológico "oscilométrico" distintivo que anuncie la presencia de la sístole y la diástole), y es menos exacta en algunos pacientes. Además, la naturaleza patentada del algoritmo dificulta considerar la oscilometría como un método de medición singular y hace de la validación del aparato (y su algoritmo) una consideración de suma importancia a la hora de seleccionar un tensiómetro automático para su uso clínico.

HTA: hipertensión arterial; MAPAC: medición automática de la presión arterial en el consultorio; PA: presión arterial.

y terapéuticos fundamentales en el campo de la HTA [17-19]. Hacen falta múltiples lecturas en el transcurso del tiempo para calcular la PA habitual, que permitan la regresión a la media y reduzcan el efecto de la bata blanca [20].

\section{Causas de medición inexacta de la presión arterial en la práctica clínica}

Lamentablemente, en la práctica clínica no suele llevarse a cabo una medición auscultatoria estandarizada [12,21], y también es raro que la lleven a cabo los estudiantes de medicina $[11,22]$. Lo más frecuente es una medición "informal" que no sigue el método estandarizado. Estas mediciones informales suelen asociarse a mayor variabilidad, con sobrestimación de la PA sistólica en 5-10 mmHg y peor correlación con los daños hipertensivos en órganos diana [10,11,15,23].

Existen múltiples causas de inexactitud, que pueden clasificarse de la siguiente manera: relacionadas con el paciente, con el procedimiento, con el tensiómetro o con el observador (cuadro 3) [24]. La auscultación mal realizada es responsable de gran cantidad de errores [10,15]. Entre los principales obstáculos a la medición estandarizada de la PA se cuentan la adherencia inadecuada a la técnica óptima, la falta de formación del observador, otras obligaciones laborales que limitan el tiempo que el observador puede dedicar a la medición y el uso de aparatos inexactos $[8,14,25]$. Entre los errores frecuentes relacionados con el observador en el ámbito clínico, cabe mencionar los siguientes: saltarse el período de reposo de 5 minutos, hablar durante el procedimiento de medición, usar un manguito de tamaño incorrecto y no tomar mediciones múltiples o bilaterales [12,23]. Las limitaciones de tiempo constituyen un motivo especialmente frecuente de medición informal, pues una lectura informal lleva solo unos 2 minutos, frente a los 8 minutos que precisa una medición estandarizada $[12,26]$. Las cifras tensionales tomadas por médicos suelen ser mayores que las registradas por enfermeras, lo cual se ha atribuido a un mayor efecto de la bata blanca [27]. Por último, el observador es responsable de realizar una medición adecuada y garantizar, en la medida de lo posible, que se eviten todas las causas potenciales de inexactitud.

\section{Ventajas de la medición automática de la presión arterial}

El uso de la medición automática de la PA se encuentra respaldado por numerosos estudios pronósticos y terapéuticos fundamentales en el campo de la HTA [17-19]. Su principal ventaja es que reduce los errores relacionados con el observador al automatizar el proceso de medición de la PA. En consecuencia, requiere menos pericia por parte del observador, no requiere de capacitación en auscultación y el observador puede centrarse en dominar menos aspectos esenciales de la medición de la PA [28]. La medición automática de la PA podría mejorar más aún la técnica de registro si hubiera disponibilidad de tensiómetros programados para tomar la PA acorde a las recomendaciones.

A modo de ejemplo cabe mencionar las instrucciones paso a paso mostradas de forma electrónica para recordarle a los observadores la técnica correcta, y las secuencias de inicio controladas automáticamente que requieran un período cronometrado de reposo antes de llevar a cabo la primera lectura. 
CUADRO 2. Elementos esenciales para llevar a cabo una medición estandarizada de la presión arterial en el consultorio

Elemento Comentario

Tensiómetro

Use un aparato calibrado (para tensiómetros aneroides) o validado clínicamente (para tensiómetros automáticos).

La columna de mercurio debe estar en cero en reposo, y completamente intacta y legible.

Los tensiómetros aneroides requieren calibración periódica.

Los tensiómetros electrónicos deben validarse mediante auscultación con esfigmomanómetro de mercurio y dos observadores en un estudio clínico independiente con un protocolo aceptado internacionalmente. Existen listas de tensiómetros validados; por ejemplo: https://bihsoc.org/bp-monitors y https:// hypertension.ca/hypertension-and-you/managing-hypertension/measuring-bloodpressure/devices (ambas en inglés).

\section{Preparación y colocación}

Los tensiómetros aneroides o las columnas de mercurio deben ser claramente visibles a la altura de los ojos.

El paciente debe permanecer en reposo sentado cómodamente en una silla en un ambiente tranquilo durante $5 \mathrm{~min}$, con la espalda apoyada en el respaldo, las piernas sin cruzar, los pies apoyados en el piso, el antebrazo apoyado y el manguito en el brazo a la altura del corazón.

\section{Manguito}

La anchura de la cámara inflable debe ser de alrededor del $40 \%$ del perímetro braquial, y su longitud, de alrededor del $80-100 \%$ del perímetro braquial. Para los tensiómetros electrónicos, seleccione el tamaño del manguito según las recomendaciones del fabricante.

Para el método auscultatorio, el borde inferior del manguito debe estar $203 \mathrm{~cm}$ por encima del pliegue del codo, y la cámara inflable debe estar centrada sobre la arteria humeral.

Para los tensiómetros electrónicos, coloque el manguito según las recomendaciones del fabricante.

\section{Procedimiento}

Para el método auscultatorio, aumente la presión rápidamente hasta $30 \mathrm{mmHg}$ por encima del valor en el cual se extingue el pulso humeral o radial, coloque el cabezal del estetoscopio sobre la arteria humeral, desinfle el manguito a un ritmo aproximado de $2 \mathrm{mmHg}$ por latido y determine las cifras sistólica (aparición de los ruidos de Korotkoff) y diastólica (desaparición de los ruidos de Korotkoff). Si los ruidos de Korotkoff persistieran hacia cero, use su punto de amortiguación como cifra de PA diastólica. Para los tensiómetros automáticos, inicie la medición según las instrucciones del aparato.

Datos tomados de [5]

Otra ventaja trascendental de la medición automática, aunque exceda el objetivo inmediato del presente documento de posición, es que permite tomar muchas mediciones de la PA fuera del consultorio, en el entorno cotidiano de cada persona. La medición de la PA fuera del consultorio abarca el registro ambulatorio durante 24 horas y el registro domiciliario de la PA, y permite evaluar mucho mejor la PA habitual porque, además de eliminar los errores relacionados con el observador, elimina también el efecto de la bata blanca (cifras tensionales altas en el consultorio, pero normales fuera de él) y detecta la HTA enmascarada (cifras tensionales normales en el consultorio, pero altas fuera de él). De hecho, debido a la existencia de estos efectos de la bata blanca y la HTA enmascarada, que afectan a $9-24 \%$ y $9-17 \%$ de las personas con y sin tratamiento antihipertensivo, respectivamente, incluso las mediciones estandarizadas de la PA tomadas de forma meticulosa en el consultorio pueden no ser representativas de la PA habitual [29]. En las directrices actuales, se recomienda confirmar el diagnóstico de HTA con mediciones de la PA fuera del consultorio y tratar la HTA enmascarada, pero no la de bata blanca [30].

El registro ambulatorio de 24 horas y el registro domiciliario de la PA son muy superiores a las mediciones en el consultorio en cuanto a la capacidad de pronosticar complicaciones cardiovasculares [31,32]. El registro de la PA durante 24 horas es
Ni el paciente ni el observador deben hablar durante todo el procedimiento de medición. El paciente debe tener la vejiga vacía y no haber comido, tomado cafeína, fumado ni realizado ninguna actividad física durante al menos 30 minutos antes de la medición.

Usar un manguito demasiado grande da lugar a lecturas erróneas bajas; usar un manguito demasiado pequeño, a lecturas erróneas altas. Las inscripciones del manguito indican claramente el perímetro braquial ideal para el tamaño del manguito.

Anote la PA con redondeo al múltiplo de 2 mmHg más cercano en el caso del método auscultatorio, o exactamente como aparezca en la pantalla si se trata de un tensiómetro automático. Evite toda preferencia por el dígito terminal (evite redondear el último dígito hacia arriba o hacia abajo a cero 0 a cinco). En la primera consulta debe tomarse la PA en ambos brazos, y el brazo con mayor PA es el que se usará para las mediciones posteriores. En cada consulta deben tomarse dos o más lecturas y calcular el promedio, que es la cifra que se usará para adoptar las decisiones clínicas. el método de referencia para diagnosticar la HTA, y el registro domiciliario de la PA es ideal para el seguimiento a largo plazo de los hipertensos tratados, especialmente cuando se combina con el manejo de casos por parte de enfermeras o farmacéuticos [33-36]. Si los recursos lo permiten, se recomienda enfáticamente utilizar ambos métodos de medición $[30,35,37,38]$. Las dos modalidades de medición fuera del consultorio requieren una técnica adecuada, y los profesionales de la salud deben comprender que el registro domiciliario de la PA necesita de la capacitación de los pacientes si pretende ser eficaz a la hora de mejorar las decisiones clínicas $[33,36,39]$.

Como ya hemos mencionado, la MAPAC permite realizar de tres a cinco mediciones secuenciales de la PA sin supervisión y calcula el promedio de forma automática [40]. La MAPAC es un subtipo de medición automática de la PA; la distinción fundamental radica en la automatización de lecturas secuenciales. En la práctica, eso significa que el observador únicamente debe iniciar la primera lectura de la secuencia; a continuación, puede irse y regresar cuando hayan terminado completamente las mediciones (en vez de permanecer en la sala para iniciar cada medición). El uso de la MAPAC, sobre todo cuando se deja al paciente solo en la sala, facilita un proceso de medición más estandarizado (p. ej., sin hablar, con múltiples mediciones automáticas tomadas de forma 

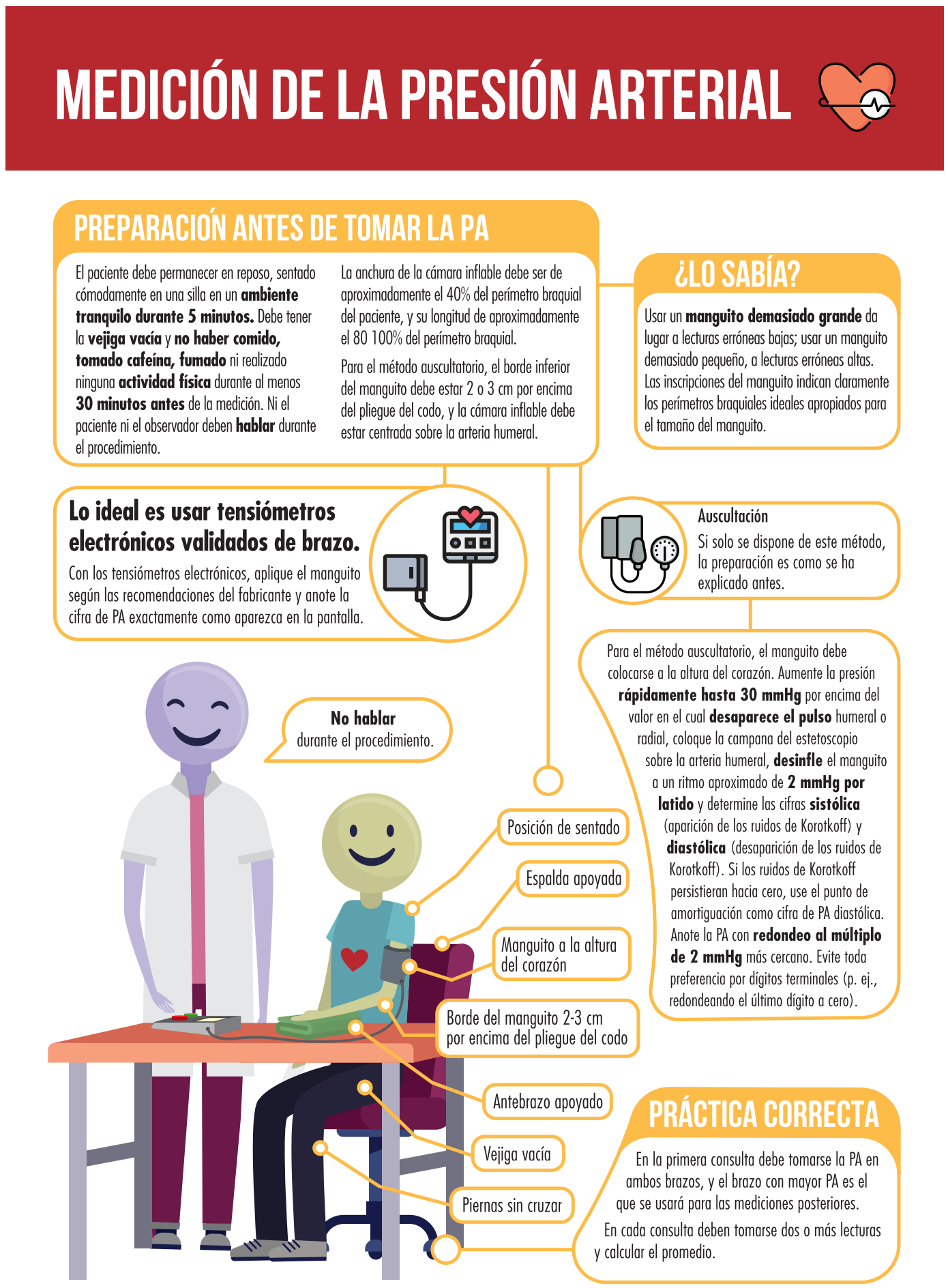

secuencial). En consecuencia, la técnica de MAPAC se asocia a menor efecto de la bata blanca [40,41,42-44]. La MAPAC requiere más espacio y más tiempo, y el costo de los tensiómetros es también entre cinco y diez veces mayor que el de un tensiómetro automático (doméstico) normal, lo cual limita también su uso en los países de ingresos bajos o medianos.
No obstante, si estos obstáculos no están presentes, debe plantearse la posibilidad de usar la MAPAC para favorecer la estandarización de las mediciones de la PA en el consultorio.

Existen algunos tensiómetros automáticos diseñados específicamente para su uso en países de ingresos bajos o medianos [45-47]. 
CUADRO 3. Fuentes principales de error al medir la presión arterial

\begin{tabular}{|c|c|c|}
\hline Fuente de error & $\begin{array}{l}\text { Intervalo de error medio } \\
\text { PA sistólica (mmHg) }\end{array}$ & $\begin{array}{l}\text { Intervalo de error medio } \\
\text { PA diastólica (mmHg) }\end{array}$ \\
\hline $\begin{array}{l}\text { Relacionado con el paciente } \\
\text { Ingestión reciente de comida } \\
\text { Consumo reciente de cafeína } \\
\text { Consumo reciente de nicotina } \\
\text { Distensión vesical } \\
\text { Efecto de la bata blanca }\end{array}$ & $\begin{array}{c}-6 \\
\text { de }+3 a+14 \\
\text { de }+3 a+25 \\
\text { de }+4 a+33 \\
\text { hasta }+26\end{array}$ & $\begin{array}{c}\text { de }-5 a-2 \\
\text { de }+2 a+13 \\
\text { de }+3 a+18 \\
\text { de }+3 a+19 \\
\text { hasta }+21\end{array}$ \\
\hline $\begin{array}{l}\text { Relacionado con el procedimiento } \\
\text { Reposo insuficiente } \\
\text { Rodillas cruzadas } \\
\text { Brazo por debajo de la altura del corazón } \\
\text { Hablar durante la medición } \\
\text { Desinflado rápido }\end{array}$ & $\begin{array}{l}\text { de }+4 a+12 \\
\text { de }+3 a+15 \\
\text { de }+4 a+23 \\
\text { de }+4 a+19 \\
\text { de }-9 a-3\end{array}$ & $\begin{array}{c}\text { de }+2 a+4 \\
\text { de }+1 a+11 \\
\text { de }+3 a+12 \\
\text { de }+5 a+14 \\
\text { de }+2 a+6\end{array}$ \\
\hline $\begin{array}{l}\text { Relacionado con el tensiómetro } \\
\text { Variabilidad del tensiómetro automático } \\
\text { Manguito demasiado pequeño } \\
\text { Manguito demasiado grande }\end{array}$ & $\begin{array}{c}\text { de }-4 a+17 \\
\text { de }+2 a+11 \\
\text { de }-4 a-1\end{array}$ & $\begin{array}{c}\text { de }-8 a+10 \\
\text { de }+2 a+7 \\
\text { de }-5 a-1\end{array}$ \\
\hline
\end{tabular}

Datos tomados de [24].

a Para optimizar la exactitud de las mediciones automáticas, use un tensiómetro validado.

\section{Exactitud de los tensiómetros automáticos}

Una cuestión importante con los tensiómetros automáticos es que muchos de ellos no han sido validados clínicamente en cuanto a la exactitud de la medición [48]. La validación clínica implica demostrar que el aparato satisface los requisitos de exactitud establecidos en las normas internacionales de medición de la PA [49]. Este proceso implica comparar, en virtud de un protocolo, múltiples mediciones frente a un patrón de referencia por técnica auscultatoria con dos observadores a ciegas. Para aumentar al máximo la exactitud, en la práctica clínica deben usarse exclusivamente tensiómetros validados [50].

En algunas personas, incluso los tensiómetros validados pueden generar mediciones de la PA que difieran sustancialmente de las cifras obtenidas por auscultación; ello puede obedecer a variaciones en el desempeño del algoritmo o en las características de la pared arterial [51,52]. Por este motivo, es deseable asegurarse de que un aparato concreto funcione de manera correcta en un paciente concreto. Por desgracia, no existe ningún consenso sobre cómo conseguir esto de un modo eficiente y factible que sea aplicable en la práctica clínica. Esta cuestión se aborda en detalle más adelante $[53,54]$.

\section{Capacitación de los observadores para mejorar la medición de la presión arterial en el consultorio}

La capacitación de los observadores se ha propuesto como una solución a la técnica deficiente de medición Y se han descrito programas de capacitación con éxito a corto plazo para mejorar la técnica; todos ellos se centran en los principios básicos de una correcta medición de la PA (cuadro 2), pero varían en cuanto al formato del curso (en línea o presencial) y su duración (desde sesiones de 30 minutos a una jornada completa)
[55-57]. Claramente se prefieren los programas más cortos e impartidos en línea debido a sus ventajas prácticas, bajo costo y escalabilidad. Más allá de la capacitación de observadores por sî sola, se han examinado también diversos programas de mejora de la calidad que combinan el uso de medición automática de la PA en el consultorio con formación de los profesionales en medición adecuada y asesoramiento sobre mejora del flujo de trabajo clínico, y se ha comprobado que aumentan el uso de la medición automática y reducen la preferencia por un dígito terminal [58]. Otros métodos propuestos son la capacitación de los pacientes para reconocer cuándo los profesionales de salud están realizando la medición de forma inadecuada y conseguir que los organismos regulatorios obliguen a usar la medición estandarizada, pero la aplicabilidad práctica de estas propuestas es dudosa $[59,60]$.

Una enfermera experimentada y capacitada en auscultación puede medir la PA ambulatoria diurna, un patrón de referencia usado con frecuencia, mejor incluso que los tensiómetros automáticos [61]. No obstante, la posibilidad de generalizar este resultado a un observador con menos experiencia, que haya asistido a un solo de capacitación, en el entorno de países de ingresos bajos o medianos, es probable que sea baja. La auscultación parece ser particularmente difícil de llevar a cabo de manera uniforme con el transcurso del tiempo. Además, la variabilidad de las cifras tensionales medidas por esta técnica entre observadores expertos, se redujo al mínimo mediante sesiones repetidas de capacitación, lo cual valida la importancia de este enfoque [62]. Sin embargo, después de cada sesión de capacitación, la variabilidad entre observadores aumentó al cabo de apenas uno o dos meses, lo cual indica que es preciso repetir la capacitación con mucha frecuencia para mantener las habilidades auscultatorias. Estos datos indican que los requisitos de capacitación son mayores a corto y largo plazo si se usa la técnica auscultatoria, lo cual hace poco factible su implementación 
generalizada, sobre todo en el entorno de los países de ingresos bajos o medianos. En consecuencia, se recomienda el uso de tensiómetros automáticos para reducir al mínimo los requisitos adicionales de capacitación.

En términos generales, la capacitación mejora las prácticas de medición de la PA en el corto plazo, y hace falta sostenerla continuamente para mantener las habilidades en el largo plazo. La frecuencia óptima de esta formación continua no está clara, aunque, como compromiso práctico y para evitar un calendario oneroso de formación continua y, al mismo tiempo, garantizar la actualización relativamente frecuente de las habilidades, se recomienda repetir la capacitación al menos una vez al año.

\section{Otros retos para optimizar la medición de la presión arterial en el consultorio en países de ingresos bajos o medianos}

En los países de ingresos bajos o medianos existen otros retos, y muchos de ellos no son fáciles de resolver. Aparecen resumidos en el cuadro 4, junto con las soluciones propuestas.

\section{Recomendaciones para optimizar el desempeño de los observadores al medir la presión arterial, así como la aplicación por las partes interesadas}

En la figura 2 se indican las recomendaciones para optimizar el desempeño de los observadores al medir la PA, así como la aplicación por las partes interesadas; estas recomendaciones se centran en realizar mediciones simplificadas y estandarizadas con aparatos validados semiautomáticos o automáticos en un entorno configurado de manera correcta, y garantizar la adecuada capacitación inicial y periódica de los observadores. Se recomienda enfáticamente la transferencia de competencias y redistribución de tareas mediante la capacitación de profesionales sanitarios no médicos, como enfermeras y agentes comunitarios de salud, para llevar a cabo las mediciones, pues libera a los médicos, escasos en relación con otros profesionales de la salud, para realizar otras tareas, y reduce también el efecto de la bata blanca $[27,63,64]$. La división de tareas por sí sola puede no mejorar el control de la PA si los consultorios están sobrecargados, el equipamiento no es confiable o no se dispone de tratamiento antihipertensivo [65]. Los planes de estudios de las escuelas de profesionales de la salud y programas formativos de posgrado deben incluir la capacitación estandarizada y el examen del desempeño en medición de la presión arterial.

\section{Orientaciones futuras}

Dada la importancia de brindar capacitación adecuada a los observadores, hacen falta programas que ayuden a estos a adquirir las habilidades necesarias para la medición correcta de la PA. Con este fin, los cursos de capacitación que proporcionen certificación en medición estandarizada, respaldados por destacadas organizaciones nacionales e internacionales activas en este campo, permitirían promover y fundamentar las mejores prácticas de medición. La Liga Mundial de Hipertensión ha elaborado recursos para ayudar a los prestadores de servicios de salud a realizar el tamizaje de la HTA [66]. Los programas de certificación en medición adecuada de la PA no deben resultar onerosos y deben ser sencillos, breves, multilingües, de bajo
CUADRO 4. Retos y posibles soluciones para optimizar la medición de la presión arterial en el consultorio en países de ingresos bajos o medianos

\begin{tabular}{lc}
\multicolumn{1}{c}{ Retos } & \multicolumn{1}{c}{ Solución propuesta } \\
\hline Falta de priorización y financiamiento & Aumento de la sensibilización y \\
de la atención de la HTA y medición & reconocimiento de la importancia y el \\
adecuada de la PA, incluida & alcance del problema. \\
la retribución del personal, el &
\end{tabular}

financiamiento programático y el equipamiento.

Escasa formación y capacitación de los observadores en medición estandarizada de la PA.

Gran volumen de trabajo y poco tiempo para realizar correctamente la medición, incluido cambio del manguito de un paciente a otro. Ausencia de un área específica en el consultorio para medir la PA.

Carencia de tensiómetros automáticos clínicamente validados, fáciles de manejar y baratos, Io cual obliga a usar el método auscultatorio.

Carencia de accesorios para los tensiómetros, como pilas y manguitos suplementarios. Ausencia de mecanismos adecuados y ecológicos para desechar las pilas gastadas. Acceso escaso o nulo al suministro eléctrico.

\section{Temperaturas ambientales} extremadamente altas en algunas regiones, que en teoría pueden afectar el desempeño de los tensiómetros.

HTA: hipertensión arterial; PA: presión arterial.
Capacitación fácilmente accesible y asequible. Uso de tensiómetros semiautomáticos o automáticos para eliminar la necesidad de habilidades auscultatorias, así como los errores resultantes de una auscultación mal realizada.

\section{Simplificación de la medición}

modificando la ergonomía del lugar de trabajo para facilitar las mejores prácticas (p. ej., disponer de un área específica para medir la PA, con una silla y superficie de apoyo para el antebrazo, aunque no sea en un espacio clínico aparte, y disponer los muebles de manera tal de optimizar la posición del paciente y el observador).

Aumento de la sensibilización y concientización sobre la necesidad de validación clínica y tensiómetros de bajo costo. Alentar a los fabricantes a que comercialicen aparatos de bajo costo tanto de consultorio como domiciliarios y ambulatorios.

La elección de tensiómetros validados semiautomáticos o de carga solar solventa los requisitos de pilas 0 suministro eléctrico. Un compromiso razonable, si únicamente se dispone de un solo tamaño de manguito, es elegir el tamaño que se considere óptimo para la mayoría de los pacientes atendidos en ese entorno clínico.

Requiere más estudio. costo (de ser posible, gratuitos), fáciles de repetir y ampliamente accesibles. Hace falta más investigación para determinar los mejores métodos de impartir la capacitación y más trabajo para establecer cómo y dónde realizarla, al igual que si debe requerirse la certificación para el desempeño de esta práctica en el ámbito de los servicios de salud.

\section{CONCLUSIONES}

Dada la enorme y creciente carga mundial de la HTA, es evidente que resulta preciso mejorar todos los aspectos de su prevención, diagnóstico, tratamiento y control [4]. Nunca se insistirá lo suficiente en la importancia de medir adecuadamente la PA para el diagnóstico y manejo óptimos de la HTA. Gran parte de los errores en la medición de la PA corresponden al observador y 11 or consiguiente, es preciso simplificar, estandarizar y automatizar las prácticas de medición, así como 
FIGURA 2. Recomendaciones para optimizar el desempeño de los observadores al medir la presión arterial en el consultorio, así como la aplicación por las partes interesadas

\begin{tabular}{|c|c|c|}
\hline \multirow{3}{*}{$\begin{array}{l}\text { Gobiernos y organismos } \\
\text { regulatorios } \\
\text { Requerir capacitación } \\
\text { de los observadores y } \\
\text { normas de } \\
\text { certificación }\end{array}$} & Recomendaciones & \multirow{7}{*}{$\begin{array}{l}\text { Consultorios y personal } \\
\text { - } \quad \begin{array}{l}\text { Requerir capacitación } \\
\text { y certificación de los } \\
\text { observadores } \\
\text { Garantizar el } \\
\text { cumplimiento de las } \\
\text { normas }\end{array} \\
\text { Revistas científicas } \\
\text { Requerir notificación } \\
\text { adecuada y aceptar } \\
\text { solo estudios que } \\
\text { usen mediciones } \\
\text { estandarizadas } \\
\text { Fabricantes } \\
\text { Promover la medición } \\
\text { estandarizada } \\
\text { Incluir instrucciones de } \\
\text { medición estandarizada } \\
\text { con los aparatos a la } \\
\text { venta }\end{array}$} \\
\hline & $\begin{array}{c}\text { Configuración de las características de medición de la PA } \\
\text { para garantizar el uso de prácticas estandarizadas } \\
\text { de medición. }\end{array}$ & \\
\hline & $\begin{array}{l}\text { División de tareas (capacitación de profesionales sanitarios no } \\
\text { médicos u otros prestadores) para medir la PA. }\end{array}$ & \\
\hline \multirow{4}{*}{$\begin{array}{l}\text { Sociedades } \\
\text { profesionales y ONG } \\
\text { - } \quad \text { Elaborar e implantar } \\
\text { normas y programas } \\
\text { de capacitación y } \\
\text { certificación } \\
\text { - } \text { Promover tales } \\
\text { programas } \\
\text { - Acreditar los } \\
\text { programas de } \\
\text { capacitación } \\
\text { existentes }\end{array}$} & $\begin{array}{c}\text { Garantizar la capacitación adecuada de los observadores, a } \\
\text { ser posible con certificación, y renovación anual } \\
\text { de la capacitación. }\end{array}$ & \\
\hline & $\begin{array}{c}\text { Medición de la PA con la técnica estandarizada } \\
\text { recomendada. Uso de manguitos del tamaño adecuado para } \\
\text { el perímetro braquial. Si no hubiera manguitos de múltiples } \\
\text { tamaños, elección del tamaño óptimo para la } \\
\text { mayoría de la población. }\end{array}$ & \\
\hline & $\begin{array}{l}\text { Uso de tensiómetros semiautomáticos o automáticos de } \\
\text { brazo validados en lugar del método auscultatorio para } \\
\text { reducir la necesidad de capacitación y optimizar la } \\
\text { medición de la PA. }\end{array}$ & \\
\hline & $\begin{array}{l}\text { Uso de tensiómetros semiautomáticos o de carga solar } \\
\text { validados si los recursos limitan el uso de tensiómetros } \\
\text { automáticos eléctricos o de pilas. }\end{array}$ & \\
\hline
\end{tabular}

ONG: organizaciones no gubernamentales; PA: presión arterial.

también garantizar la adecuada formación, capacitación y certificación de los observadores. Incluso estas recomendaciones relativamente directas pueden ser difíciles de implementar, pero tienen el potencial de mejorar de manera notable la detección y el manejo de la HTA en todo el mundo. Dada la importancia de evaluar de forma exacta la PA, y la escasa repercusión de los esfuerzos anteriores por capacitar a los profesionales de la salud, debe considerarse la posibilidad de establecer la certificación periódica en evaluación de la PA.

Agradecimientos. Respaldan el presente consenso la Liga Mundial de Hipertensión, la Sociedad ARTERY, la Asociación Médica Estadounidense (AMA), la Asociación Estadounidense del Corazón (AHA), Hipertensión Canadá, la iniciativa Resolve to Save Lives, y el Grupo de trabajo de la Sociedad Europea de Hipertensión sobre registro de la presión arterial y variabilidad cardiovascular.

Conflictos de intereses. R.P. es cofundador de una empresa emergente de medición de la presión arterial, $\mathrm{mmHg}$ Inc, que en la actualidad no tiene ningún producto comercializado. N.R.C.C. fue asesor remunerado de la Fundación Novartis (2016 - 2017) en apoyo de su programa para mejorar el control de la hipertensión en países de ingresos bajos o medianos, lo cual incluyó gastos de viaje para visitar los centros y un contrato para elaborar una encuesta; además, ha prestado asesoramiento consultivo remunerado para Midway Corporation (2017) sobre evaluación exacta de la presión arterial. M.K.R. es vicepresidente de Mejora de Resultados en Salud en la Asociación Médica Estadounidense. G.W. es director de Analítica de Resultados en la Asociación Médica Estadounidense. G.S. preside el Grupo de trabajo de la Sociedad Europea de Hipertensión sobre registro de la presión arterial, es vocal del Comité de Esfigmomanómetros de la ISO, y ha realizado estudios de validación para diversos fabricantes de tecnologías de medición de la presión arterial y asesorado a los fabricantes sobre desarrollo de aparatos y de programas informáticos. J.K.C. fue el anterior presidente de la Sociedad ARTERY. A.E.S. ha recibido honorarios de Novartis y Omron como ponente por conferencias científicas sobre presión arterial y evaluación de riesgos cardiovasculares, y de Servier por disertar sobre concientización en medición de la presión arterial; además, es asesora remunerada de Abbott Pharmaceuticals sobre medicamentos antihipertensores y presidente de la Sociedad Internacional de Hipertensión. El resto de los autores no revela ningún conflicto de intereses.

\section{REFERENCIAS}

1. Global Burden of Disease Risk Factor Collaborators. Global, regional, and national comparative risk assessment of 84 behavioural, environmental and occupational, and metabolic risks or clusters of risks for 195 countries and territories, 1990-2017: a systematic analysis for the Global Burden of Disease Study, 2017. Lancet 2018; 392: 1923-1994.
2. Mills KT, Bundy JD, Kelly TN, Reed JE, Kearney PM, Reynolds K, et al. Global disparities of hypertension prevalence and control: a systematic analysis of population-based studies from 90 countries. Circulation 2016; 134: 441-450.

3. Benjamin EJ, Virani SS, Callaway CW, Chamberlain AM, Chang AR, Cheng S, et al.; American Heart Association Council on 
Epidemiology and Prevention Statistics Committee and Stroke Statistics Subcommittee. Heart disease and stroke statistics - 2018 update: a report from the American Heart Association. Circulation 2018; 137: e67-e492.

4. Olsen MH, Angell SY, Asma S, Boutouyrie P, Burger D, Chirinos JA, et al. A call to action and a lifecourse strategy to address the global burden of raised blood pressure on current and future generations: the Lancet Commission on hypertension. Lancet 2016; 388: 2665-2712.

5. Pickering TG, Hall JE, Appel LJ, Falkner B, J G, Hill MN, et al. Recommendations for blood pressure measurement in humans and experimental animals: Part 1: blood pressure measurement in humans: a statement for professionals from the subcommittee of professional and public education of the American Heart Association Council on High Blood Pressure Research. Circulation 2005; 111: 697-716.

6. Joffres MR, Campbell NRC, Manns B, Tu K. Estimate of the benefits of a population-based reduction in dietary sodium additives on hypertension and its related healthcare costs in Canada. Can J Cardiol 2007; 23: 437-443.

7. Raven PB, Chapleau MW. Blood pressure regulation XI: overview and future research directions. Eur I Appl Physiol 2014; 114: 579—586.

8. Pickering TG, Gerin W, Schwartz JE, Spruill TM, Davidson KW. Franz Volhard lecture: should doctors still measure blood pressure? The missing patients with masked hypertension. J Hypertens 2008; 26: 2259-2267.

9. Riva-Rocci S, Zanchetti A, Mancia G. A new sphygmomanometer. Sphygmomanometric technique. J Hypertens 1996; 14: 1-12.

10. Campbell NR, Myers MG, McKay DW. Is usual measurement of blood pressure meaningful? Blood Press Monit 1999; 4:71-76.

11. McKay DW, Raju MK, Campbell NR. Assessment of blood pressure measuring techniques. Med Educ 1992; 26:208-212.

12. Ray GM, Nawarskas JJ, Anderson JR. Blood pressure monitoring technique impacts hypertension treatment. J Gen Intern Med 2011; 27:623-629.

13. O'Brien E, Stergiou GS. The pursuit of accurate blood pressure measurement: a 35-year travail. J Clin Hypertens (Greenwich) 2017; 19:746-752.

14. O'Brien E, Dolan E, Stergiou GS. Achieving reliable blood pressure measurements in clinical practice: It's time to meet the challenge. J Clin Hypertens 2018; 20:1084-1088.

15. Campbell NRC, Culleton BW, McKay DW. Misclassification of blood pressure by usual measurement in ambulatory physician practices. Am J Hypertens 2005; 18 (12 Pt 1): 1522-1527.

16. Alpert BS, Quinn D, Gallick D. Oscillometric blood pressure: a review for clinicians. J Am Soc Hypertens 2014; 8:930-938.

17. Ettehad D, Emdin CA, Kiran A, Anderson SG, Callender T, Emberson J, et al. Blood pressure lowering for prevention of cardiovascular disease and death: a systematic review and meta-analysis. Lancet 2015; 387:957-967.

18. Chen Y, Lei L, Wang JG. Methods of blood pressure assessment used in milestone hypertension trials. Pulse (Basel) 2018; 6: 112-123.

19. Giorgini P, Weder AB, Jackson EA, Brook RD. A review of blood pressure measurement protocols among hypertension trials: implications for 'evidence-based' clinical practice. J Am Soc Hypertens 2014; 8: 670-676.

20. Brueren MM, Petri H, Van Weel C, Van Ree JW. How many measurements are necessary in diagnosing mild to moderate hypertension. Fam Pract 1997; 14: 130-135.

21. Sebo P, Pechere-Bertschi A, Herrmann FR, Haller DM, Bovier P. Blood pressure measurements are unreliable to diagnose hypertension in primary care. J Hypertens 2014; 32: 509-517.

22. Rakotz MK, Townsend RR, Yang J, Alpert BS, Heneghan KA, Wynia $\mathrm{M}$, et al. Medical students and measuring blood pressure: results from the American Medical Association Blood Pressure Check Challenge. J Clin Hypertens (Greenwich) 2017; 19: 614-619.

23. Minor DS, Butler KR, Artman KL, Adair C, Wang W, McNair V, et al. Evaluation of blood pressure measurement and agreement in an academic health sciences center. J Clin Hypertens (Greenwich) 2012; 14: 222-227.

24. Kallioinen N, Hill A, Horswill MS, Ward HE, Watson MO. Sources of inaccuracy in the measurement of adult patients' resting blood pressure in clinical settings. J Hypertens 2017; 35: 421-441.
25. O'Brien E, Asmar R, Beilin L, Imai Y, Mancia G, Mengden T, et al.; European Society of Hypertension Working Group on Blood Pressure Monitoring. Practice guidelines of the European Society of Hypertension for clinic, ambulatory and self blood pressure measurement. J Hypertens 2005; 23: 697-701.

26. Stergiou GS, Kyriakoulis KG, Kollias A. Office blood pressure measurement types: different methodology-different clinical conclusions. J Clin Hypertens (Greenwich) 2018; 20: 1683-1685.

27. Clark CE, Horvath IA, Taylor RS, Campbell JL. Doctors record higher blood pressures than nurses: systematic review and meta-analysis. Br J Gen Pract 2014; 64: e223-e232.

28. Campbell NR, Berbari AE, Cloutier L, Gelfer M, Kenerson JG, Khalsa TK, et al. Policy statement of the world hypertension league on noninvasive blood pressure measurement devices and blood pressure measurement in the clinical or community setting. J Clin Hypertens 2014; 16: 320-322.

29. Fagard RH, Cornelissen VA. Incidence of cardiovascular events in white-coat, masked and sustained hypertension versus true normotension: a meta-analysis. J Hypertens 2007; 25: 2193-2198.

30. Williams B, Mancia G, Spiering W, Agabiti Rosei E, Azizi M, Burnier M, et al. 2018 ESC/ESH guidelines for the management of arterial hypertension: The Task Force for the management of arterial hypertension of the European Society of Cardiology and the European Society of Hypertension. J Hypertens 2018; 36: 1953-2041.

31. O’Brien E, Parati G, Stergiou G, Asmar R, Beilin L, Bilo G, et al.; European Society of Hypertension Working Group on Blood Pressure Monitoring. European Society of Hypertension position paper on ambulatory blood pressure monitoring. J Hypertens 2013; 31: 1731-1768.

32. Stergiou GS, Bliziotis IA. Home blood pressure monitoring in the diagnosis and treatment of hypertension: a systematic review. Am J Hypertens 2009; 24: 123-134.

33. Cloutier L, Daskalopoulou S, Padwal RS, Lamarre-Clich M, Bolli P, McLean D, et al. A new algorithm for the diagnosis of hypertension in Canada. Can J Cardiol 2015; 31: 620-630.

34. Duan Y, Xie Z, Dong F, Wu Z, Lin Z, Sun N, et al. Effectiveness of home blood pressure telemonitoring: a systematic review and meta analysis of randomised controlled studies. J Hum Hypertens 2017; 31: 427-437.

35. National Institute for Health and Care Excellence. Hypertension in adults: diagnosis and management. Clinical guideline CG127. 2011 (actualización del 2016). https:/ / www.nice.org.uk/guidance/ cg127 [consultado el 12 de octubre del 2018]

36. Parati G, Stergiou G, O’Brien E, Asmar R, Beilin L, Bilo G, et al. European Society of Hypertension practice guidelines for ambulatory blood pressure monitoring. J Hypertens 2014; 32: 1359-1366.

37. Nerenberg KA, Zarnke KB, Leung AA, Dasgupta K, Butalia S, McBrienK, et al., Hypertension Canada. Hypertension Canada's 2018 Guidelines for Diagnosis, Risk Assessment, Prevention, and Treatment of Hypertension in Adults and Children. Hypertension Canada's 2018 guidelines for diagnosis, risk assessment, prevention, and treatment of hypertension in adults and children. Can J Cardiol 2018; 34: 506-525.

38. US Preventive Services Task Force. Screening for high blood pressure: U.S. Preventive Services Task Force recommendation statement. Ann Intern Med 2015; 163: 778-786.

39. Milot JP, Birnbaum L, Larochelle P, Wistaff R, Laskine M, Van Nguyen $\mathrm{P}$, et al. Unreliability of home blood pressure measurement and the effect of a patient-oriented intervention. Can J Cardiol 2015; 31: 658-663.

40. Myers MG. Eliminating the human factor in office blood pressure measurement. J Clin Hypertens (Greenwich) 2014; 16: 83-86.

41. Roerecke M, Kaczorowski J, Myers MG. Comparing automated office blood pressure readings with other methods of blood pressure measurement for identifying patients with possible hypertension. JAMA Intern Med 2019; 179: 351-362.

42. Jegatheswaran J, Ruzicka M, Hiremath S, Edwards C. Are automated blood pressure monitors comparable to ambulatory blood pressure monitors? A systematic review and meta-analysis. Can J Cardiol 2017; 33: 644-652.

43. Ringrose JS, Cena J, Ip S, Morales F, Hamilton P, Padwal R. Comparability of automated office blood pressure to daytime 24-Hour ambulatory blood pressure. Can J Cardiol 2018; 34: 61-65. 
44. Drawz PE, Pajewski NM, Bates JT, Bello NA. Effect of intensive versus standard clinic-based hypertension management on ambulatory blood pressure: novelty and significance. Hypertension 2017; 69: $42-50$

45. De Greeff A, Nathan H, Stafford N, Liu B, Shennan AH. Development of an accurate oscillometric blood pressure device for low resource settings. Blood Press Monit 2008; 13: 342-348.

46. Baker EC, Hezelgrave N, Magesa SM, Edmonds S, De Greeff A, Shennan A. Introduction of automated blood pressure devices intended for a low resource setting in rural Tanzania. Trop Doct 2012; 42: 101-103.

47. Parati G, Kilama MO, Faini A, Facelli E, Ochen K, Opira C, et al. A new solar-powered blood pressure measuring device for lowresource settings. Hypertension 2010; 56: 1047-1053.

48. O'Brien E, Stergiou GS, Turner MJ. The quest for accuracy of blood pressure measuring devices. J Clin Hypertens (Greenwich) 2018; 20: 1092-1095.

49. Association for the Advancement of Medical Instrumentation. ANSI/AAMI/ISO 81060-2:2013 noninvasive sphygmomanometers - part 2: clinical investigation of automated measurement type. 2013. Association for the Advancement of Medical Instrumentation. Arlington (Virginia), 2013. https: / / www.aami.org/ [consultado el 10 de agosto del 2013].

50. Jung MH, Kim GH, Kim JH, Moon KW, Yoo KD, Rho TH, et al. Reliability of home blood pressure monitoring: in the context of validation and accuracy. Blood Press Monit 2015; 20: 215-220.

51. Stergiou GS, Lourida P, Tzamouranis D, Baibas NM. Unreliable oscillometric blood pressure measurement: prevalence, repeatability and characteristics of the phenomenon. J Hum Hypertens 2009; 23: 794-800.

52. Padwal R, Jalali A, McLean D, Anwar S, Smith K, Raggi P, et al. Accuracy of oscillometric blood pressure algorithms in healthy adults and in adults with cardiovascular risk factors. Blood Press Monit 2018; 24: 33-37.

53. Cohen JB, Padwal RS, Gutkin M, Green BB, Bloch MJ, Germino FW, et al. History and justification of a national blood pressure measurement validated device listing. Hypertension 2019; 73: 258-264.

54. Eguchi K, Kuruvilla S, Ishikawa J, Schwartz JE, Pickering TG. A novel and simple protocol for the validation of home blood pressure monitors in clinical practice. Blood Press Monit 2012; 17: 210-213.

55. Grim CM, Grim CE. A curriculum for the training and certification of blood pressure measurement for healthcare providers. Can J Cardiol 1995; 11 (supl H): 38H-42H.

56. Block L, Flynn SJ, Cooper LA, Lentz C, Hull T, Dietz KB, et al. Promoting sustainability in quality improvement: an evaluation of a web-based continuing education program in blood pressure measurement. BMC Fam Pract 2018; 19:13.
57. Dickson BK, Hajjar I. Blood pressure measurement education and evaluation program improves measurement accuracy in community based nurses: a pilot study. J Am Acad Nurse Pract 2007; 19: 93-102.

58. Boonyasai RT, Carson KA, Marsteller JA, Dietz KB, Noronha GJ, Hsu YJ, et al. A bundled quality improvement program to standardize clinical blood pressure measurement in primary care. J Clin Hypertens 2017; 20: 324-333.

59. Appel LJ, Miller ER, Charleston J. Improving the measurement of blood pressure: is it time for regulated standards. Ann Intern Med 2011; 154: 838-840.

60. Umscheid CA, Townsend RR. Is it time for a blood pressure measurement 'bundle'? J Gen Intern Med 2012; 27: 615-617.

61. Graves JW, Grossardt BR, Gullerud RE, Bailey KR, Feldstein J. The trained observer better predicts daytime ABPM diastolic blood pressure in hypertensive patients than does an automated (Omron) device. Blood Pres Monit 2006; 11: 53-58.

62. Bruce NG, Shaper AG, Walker M, Wannamethee G. Observer bias in blood pressure studies. J Hypertens 1988; 6: 375-380.

63. Organización Mundial de la Salud. Global Health Observatory (GHO) data. Density of physicians (total number per 100 population, latest available year). https://www.who.int/gho/health_workforce/physicians_density/en/ [consultado el 4 de noviembre del 2018].

64. Organización Panamericana de la Salud. Paquete técnico HEARTS para el manejo de las enfermedades cardiovasculares en la atención primaria de salud: trabajo basado en equipos multidisciplinarios. Washington, DC: OPS, 2019 (WHO/NMH/NVI/18.4). Licencia: CC BY-NC-SA 3.0 IGO.

65. Goudge J, Chirwa T, Eldridge S, Gómez-Olivé FX, Kabudula C, Limbani F, et al. Can lay health workers support the management of hypertension? Findings of a cluster randomised trial in South Africa. BMJ Glob Health 2018; 3: e000577-e12.

66. Mangat BK, Campbell N, Mohan S, Niebylski ML, Khalsa TK, Berbari AE, et al. Resources for blood pressure screening programs in low resource settings: a guide from the World Hypertension League. J Clin Hypertens (Greenwich) 2015; 17: 418-420.

Manuscrito original (inglés) recibido: 9 de febrero del 2019; revisado: 6 de marzo del 2019; aceptado: 11 de marzo del 2019. 


\section{Optimizing observer performance of clinic blood Pressure measurement: a position statement from the Lancet Commission on Hypertension Group}

ABSTRACT High blood pressure (BP) is a highly prevalent modifiable cause of cardiovascular disease, stroke, and death. Accurate BP measurement is critical, given that a $5-\mathrm{mmHg}$ measurement error may lead to incorrect hypertension status classification in 84 million individuals worldwide. This position statement summarizes procedures for optimizing observer performance in clinic BP measurement, with special attention given to low-tomiddle-income settings, where resource limitations, heavy workloads, time constraints, and lack of electrical power make measurement more challenging. Many measurement errors can be minimized by appropriate patient preparation and standardized techniques. Validated semi-automated/automated upper arm cuff devices should be used instead of auscultation to simplify measurement and prevent observer error. Task sharing, creating a dedicated measurement workstation, and using semi-automated or solar-charged devices may help. Ensuring observer training, and periodic re-training, is critical. Low-cost, easily accessible certification programs should be considered to facilitate best BP measurement practice.

Keywords $\quad$ Arterial pressure; measurement equipment; consensus; global health; hypertension; oscillometry.

\section{Otimização do desempenho do observador na medição clínica da pressão arterial: posicionamento do Grupo da Lancet Commission on Hypertension}

RESUMO A hipertensão é uma causa altamente prevalente de doença cardiovascular, acidente vascular cerebral e morte. A medição precisa da pressão arterial (PA) é um aspecto crítico, uma vez que erros de mensuração da ordem de $5 \mathrm{mmHg}$ podem levar a uma classificação incorreta do status de hipertensão em 84 milhões de pessoas em todo o mundo. O presente posicionamento resume os procedimentos para otimizar o desempenho do observador (o indivíduo responsável pela mensuração da PA) na mensuração clínica da PA, com atenção especial para contextos de baixa a média renda, onde recursos limitados, cargas de trabalho pesadas, restrições de tempo e falta de energia elétrica tornam mais desafiadora a tarefa de medir a PA. Muitos erros de mensuração podem ser minimizados pela preparação adequada do paciente e pelo uso de técnicas padronizadas. Para simplificar a mensuração e evitar erros do observador, devem-se utilizar dispositivos semiautomatizados ou automatizados validados, com manguito para braço, ao invés de auscultação. O compartilhamento de tarefas, a criação de uma estação de trabalho dedicada à mensuração e o uso de dispositivos semiautomatizados ou com carga solar podem ajudar. É essencial que seja assegurado o treinamento e retreinamento periódico do observador. Programas de certificação de baixo custo e de fácil acesso devem ser considerados para facilitar a adoção das melhores práticas na mensuração da PA.

Palavras-chave Pressão arterial; equipamentos de medição; consenso; saúde global; hipertensão; oscilometria. 